\title{
Corrections to Gravity due to a Sol Manifold Extra Dimensional Space
}

\author{
V.K.Oikonomou* \\ Dept. of Theoretical Physics Aristotle University of Thessaloniki, \\ Thessaloniki 54124 Greece
}

December 14, 2018

\begin{abstract}
The corrections to the gravitational potential due to a Sol extra dimensional compact manifold, denoted as $M_{A}^{3}$, are studied. The total spacetime is of the form $M^{4} \times M_{A}^{3}$. The range of the Sol corrections is investigated and compared to the range of the $T^{3}$ corrections.
\end{abstract}

\section{Introduction}

It has been nearly ten years since the originating papers studying Tev scale extra dimensions [1] appeared. After that, the phenomenological implications of extra dimensional models were extensively studied. Many of these studies are concentrated on the modifications caused on the gravitational potential due to extra dimensions [4, 7]. In general the modifications are of Yukawa type, differing only in their strength and range. In this letter we shall investigate the modification of the gravitational potential caused by a Sol extra dimensional manifold. Sol manifolds are compact and orientable three dimensional manifolds, denoted as $M_{A}^{3}$. In the following chapters we shall exploit the Sol manifolds geometric structure and the study of the Laplace equation on such manifolds. After that we compare the range of the corrections to the gravitational potential due to Sol manifolds, with the corresponding range of the corrections caused by a $T^{3}$ extra dimensional manifold.

\section{Sol Manifold Geometry and Topology}

Sol manifolds are one of Thurston's 8 three dimensional geometries [2] (geometric structures). The geometrization conjecture is an approach to the geometry of three dimensional

*voiko@physics.auth.gr 
manifolds through general topological arguments. Actually one starts with the fact that three dimensional manifolds compose from 2-spheres or torii surfaces. According to the properties and the details of the topological gluing map of the above with $R$ or $S^{1}$, the resulting manifolds acquire homogeneous metrics (at least locally). Sol manifolds are described by one type of the 8 different homogeneous metrics.

Sol manifolds result from $S L(2, Z)$ stiffennings of torus bundles over the circle. More elaborately a theorem [6] states: given $\mathrm{M}$ a total bundle space of a $T^{2}$ bundle over $S^{1}$ with gluing map $\phi$ and let $A \epsilon G L(2, Z)$ represent the automorphism of the fundamental group of the torus $T^{2}$ induced by the gluing map $\phi$. Then the total bundle space admits a Sol geometric structure if $A$ is hyperbolic, an $E^{3}$ structure if $A$ is periodic and finally a Nil structure otherwise. $A$ is hyperbolic and gives rise to an orientable manifold if $|\operatorname{Tr} A|>2$ and we shall dwell on this choice. More formally, the Sol structure arises from the $S L(2, Z)$ stiffening of the mapping torus of a torus diffeomorphism $\phi[2]$.

Before proceeding let us make a comment on the structure of $A$. Two different hyperbolic gluings with $A_{1} \neq A_{2}$, give rise to different geometric Sol structures. For $A$ we will use the matrix representation of the form

$$
A=\left(\begin{array}{cc}
0 & 1 \\
-1 & n
\end{array}\right)
$$

so the different classes of Sol geometric structures are classified by the integer $n$, with $|n|=|\operatorname{Tr} A|>2$. However this classification is not the only one existing [8], nevertheless this will do the classification properly in our case. Also we shall take into account only the positive eigenvalues of $A$.

Let us be more quantitative on the Sol manifold analysis. Consider the manifold $T^{2} \times \mathbb{R}$ described by the periodic coordinates $(x, y)$ for the torus, defined modulo $R$ (the radius of the compact dimensions) and $z \epsilon(-\infty, \infty)$ be a coordinate of $\mathbb{R}$. The combined action of the torus mapping through the hyperbolic gluing map $A$ diffeomorphism (which we denote as $\bar{\Gamma})$ is :

$$
\bar{\Gamma}:\left(\begin{array}{c}
x \\
y \\
z
\end{array}\right) \rightarrow\left(\begin{array}{c}
a_{11} x+a_{12} y \\
a_{21} x+a_{22} y \\
z+2 \pi R
\end{array}\right)
$$

We give a general notation for the matrix $A$, of the form

$$
A=\left(\begin{array}{ll}
a_{11} & a_{12} \\
a_{21} & a_{22}
\end{array}\right)
$$

but in practise we shall use the form of relation (11). As we described above, the Sol manifold, which is denoted as $M_{A}^{3}$ is the quotient of $T^{2} \times \mathbb{R}$ by the action of $\bar{\Gamma}$, that is $M_{A}^{3} \equiv T^{2} \times \mathbb{R} / \bar{\Gamma}$. It is obvious that it is a total torus bundle over $S^{1}$, with $T^{2}$ the fiber, $S^{1}$ the base space and $A$ the hyperbolic gluing map of the torus fibers. We shall describe the Sol manifolds for which the eigenvalues $\lambda$ of $A$ are positive. For the case of (1) the characteristic polynomial of the matrix $A$ reads:

$$
\lambda^{2}-\operatorname{Tr} A \lambda+1=0
$$


or equivalently:

$$
\lambda^{2}-n \lambda+1=0
$$

The solutions to equation (5) are $\lambda$ and $\lambda^{-1}$ with $\lambda+\lambda^{-1}=n=\operatorname{Tr} A$. Also the discriminant of $A$ is:

$$
D=\left(a_{11}+a_{22}\right)^{2}-4
$$

which in our case reads:

$$
D=\left(\lambda-\lambda^{-1}\right)^{2}
$$

In this letter we shall make use of another coordinate system $(u, v, z)$ on the Sol manifold. The $(u, v)$ are linear coordinates of the torus fibres related to an eigenbasis of the hyperbolic map $A$. These coordinates correspond to a rotated torus lattice. The action of $\bar{\Gamma}$ in the new coordinate system now reads:

$$
\bar{\Gamma}:\left(\begin{array}{c}
u \\
v \\
z
\end{array}\right) \rightarrow\left(\begin{array}{c}
\lambda u \\
\lambda^{-1} v \\
z+2 \pi R
\end{array}\right)
$$

The original lattice was orthogonal while the new torus lattice is not. If $e_{u}$ and $e_{v}$ are the basis of the lattice after the action of $\bar{\Gamma}$ identification, then $\left(e_{u}, e_{v}\right)=\left|e_{u}\right|\left|e_{v}\right| \cos \theta$ (the basis of the new lattice coincide with the eigenvectors of $A$ corresponding to the eigenvalues $\lambda$ and $\lambda^{-1}$ ). Also the coordinates of the fibers $T^{2}$ are not periodic anymore. So in order two pairs $\left(u_{1}, v_{1}\right)$ and $\left(u_{2}, v_{2}\right)$ define the same point on the torus lattice, the following must hold:

$$
\left(u_{1}-u_{2}, v_{1}-v_{2}\right)=k e_{u}+m e_{v}
$$

with $k, m$ integers and $e_{u}, e_{v}$ defined previously.

\section{Riemannian Sol group invariant metric on Sol manifolds}

The Riemannian metric on Sol manifolds comes from the metric on the universal covering of $M_{A}^{3}$. The group invariant metric on the universal covering is a Sol group invariant metric. The Sol group is a three dimensional solvable Lie group homeomorphic to $\mathbb{R}^{3}$ and can be realized as the matrix:

$$
\left(\begin{array}{ccc}
e^{z \ln \lambda} & 0 & u \\
0 & e^{-z \ln \lambda} & v \\
0 & 0 & 1
\end{array}\right)
$$

Finding the invariant metric on the universal covering of $M_{A}^{3}$ then using the relaxation method [6] we can find the following class of metrics of Sol manifolds (invariant under the group (10)):

$$
\mathrm{d} s^{2}=E e^{2 z \ln \lambda} \mathrm{d} u^{2}+2 F \mathrm{~d} u \mathrm{~d} v+G e^{-2 z \ln \lambda} \mathrm{d} v^{2}+\mathrm{d} z^{2}
$$

In the following we shall use the metric (11) in order to compute the Laplace-Beltrami operator on $M_{A}^{3}$. 


\section{Newton's law and extra dimensions}

The main purpose of this paper is to examine the corrections to the gravitational potential caused by an extra dimensional Sol manifold. The spacetime manifold is of the form $M^{4} \times M_{A}^{3}$. In this section we review the general technique to obtain these corrections. The presentation is based on reference [4]. The techniques that will be presented are applied to compact manifolds, since this is the case of $M_{A}^{3}$. A generalization to non-compact manifolds can be found in [5].

Consider a spacetime of the form $M^{4} \times M^{n}$, with $M^{n}$ an n-dimensional compact manifold and $M^{4}$ the four dimensional Minkowski spacetime. Suppose there exist a complete set of orthogonal harmonic functions on $M^{4}, \Psi_{m}$, satisfying the orthogonality condition:

$$
\int_{M^{n}} \Psi_{n}(x) \Psi_{m}^{*}(x)=\delta_{n, m}
$$

and the completeness relation:

$$
\sum_{m} \Psi_{m}(x) \Psi_{m}^{*}\left(x^{\prime}\right)=\delta^{(n)}\left(x-x^{\prime}\right)
$$

The functions $\Psi_{m}$ are eigenfunctions of the $n$-dimensional Laplace-Beltrami operator $\Delta_{n}$ of the manifold $M^{n}$, with eigenvalues $\mu_{m}^{2}$ :

$$
-\Delta_{n} \Psi_{m}=\mu_{m}^{2} \Psi_{m}
$$

The gravitational potential $V_{n+4}$ satisfies the Poisson equation in $n+3$ spatial dimensions, when the Newtonian limit is taken:

$$
\Delta_{n+3} V_{n+4}=(n+1) \Omega_{n+2} G_{n+4} M \delta^{(n+3)}(x)
$$

with $M$, the mass of the system, $G_{n+4}$ the Newton constant in $n+4$ dimensions and

$$
\Omega_{n+2}=\frac{2 \pi^{\frac{n+3}{2}}}{\Gamma\left(\frac{n+3}{2}\right)}
$$

Equation (15) corresponds to the case of large compact radius limit and has the solution:

$$
V_{n+4}=-\frac{G_{n+4} M}{r_{n}^{n+1}}
$$

In the case of our interest, where the compact dimensions have small lengths, we find the harmonic expansion of $V_{n+4}$ in terms of the eigenfunctions of the product space $M^{4} \times M^{n}$, which reads:

$$
V_{n+4}=\sum_{m} \Phi_{m}(r) \Psi_{m}(x)
$$

with $r$ denoting the coordinates of $M^{4}$ and $x$ denoting the coordinates of $M^{n}$. Consequently, the $\Phi_{m}$ obey:

$$
\Delta_{3} \Phi_{m}-\mu_{m}^{2} \Phi_{m}=(n+1) \Omega_{n+2} \Psi_{m}^{*}(0) G_{n+4} M \delta^{3}(x)
$$


with solution:

$$
\Phi_{m}(r)=-\frac{\Omega_{n} G_{n+4} M \Psi_{m}^{*}(0)}{2} \frac{e^{-\left|\mu_{m}\right| r}}{r}
$$

Finally the gravitational potential is written as:

$$
V_{n+4}=-\frac{\Omega_{n} G_{n+4} M}{2 r} \sum_{m} \Psi_{m}^{*}(0) \Psi_{m}(x) e^{-\left|\mu_{m}\right| r}
$$

Since all point particles in the four dimensional spacetime have no dependence on the internal compact space $M^{n}$, we can take $x=0$ in (21) to obtain the four dimensional gravitational potential:

$$
V_{4}=-\frac{G_{4} M}{r} \sum_{m} \Psi_{m}^{*}(0) \Psi_{m}(0) e^{-\left|\mu_{m}\right| r}
$$

which is valid for large values of $r$, compared to the lengths of the compact dimensions.

In the above general result of relation (22) we shall apply the eigenfunctions and eigenvalues of Sol manifolds. For the eigenvalues we shall take the first two smallest eigenvalues (and their corresponding eigenfunctions) since larger eigenvalues are exponentially suppressed.

\section{Sol Manifold modification of Newton's Law}

In order to compute corrections to the gravitational potential we must solve equation (14) for the case of Sol manifold $M_{A}^{3}$. A much more elaborate analysis of this chapter can be found in [3] We shall use the $(u, v, z)$ coordinates we introduced previously. The Laplace-Beltrami operator for the manifold $M_{A}^{3}$ is:

$$
\Delta=E e^{2 z \ln \lambda} \frac{\partial^{2}}{\partial u^{2}}+2 F \frac{\partial^{2}}{\partial u \partial v}+G e^{-2 z \ln \lambda} \frac{\partial^{2}}{\partial v^{2}}+\frac{\partial^{2}}{\partial z^{2}}
$$

which stems from the Riemannian metric (11). As usual $E=\left|e_{u}\right|^{2}, F=\left|e_{v}\right|^{2}$ and $G=\left|\left(e_{u}, e_{v}\right)\right|$, where $e_{u}$ and $e_{v}$ the basis of the $T^{2}$ lattice. Thus we must solve

$$
-\Delta \psi=E \psi
$$

Since the coefficients of $\Delta$ depend on $z$, the form of the solutions of (24) are of the form:

$$
\Psi_{\gamma}(u, v, z)=e^{2 \pi i(\gamma, w)} f(z)
$$

where $\gamma$ is a vector of the dual lattice of $T^{2}$ and $w$ is a vector of the $T^{2}$ lattice. Let us note that the vector of the dual lattice $\gamma$ can be written

$$
\gamma=\frac{m}{R} e_{u}^{*}+\frac{n}{R} e_{v}^{*}
$$

with $e_{u}^{*}$ and $e_{u}^{*}$ the vector basis of the dual $T^{2}$ lattice. The vector of the $T^{2}$ lattice can be written:

$$
w=u e_{u}+v e_{v}
$$


The scalar product of $w$ and $\gamma$ is identified with the usual product between the reciprocal and the Bravais lattice. Now we shall use a proposition of reference [3], which says that a function $\Psi=e^{2 \pi i(\gamma, w)} f(z)$ satisfies equation (24) if and only if $f(z)$ satisfies the modified Mathieu equation:

$$
\left(-\frac{d^{2}}{d z^{2}}+|\nu(\gamma)| \cosh 2 \mu(z+\alpha(\gamma))\right) f(z)=\Lambda f(z)
$$

with $\mu=\ln \lambda, \nu(\gamma)=8 \pi^{2} C Q_{A^{*}}(\gamma)$ and $\alpha(\gamma)=\frac{\ln \left(\sqrt{\left.\frac{E}{G} \frac{\left|\left(\gamma, e_{u}\right)\right|}{\left(\gamma, e_{v}\right) \mid}\right)}\right.}{2 \ln \lambda}$. Also

$$
C=\frac{1}{\sqrt{D} \operatorname{Vol}\left(M_{A}^{3}\right) \sin \theta}
$$

The volume of the Sol manifold, $\operatorname{Vol}\left(M_{A}^{3}\right)$, equals the volume of the total bundle space $T^{2} \times S^{1}$. In the above $D$ is the discriminant of the matrix $A$ defined in relation (7) and $Q_{A^{*}}(\gamma)$ is the quadratic form (see [3]) corresponding to the matrix $A^{*}$ acting to the dual lattice of $T^{2}$, with $Q_{A^{*}}(\gamma)=\left(\gamma, e_{u}\right)\left(\gamma, e_{v}\right)\left(\lambda-\lambda^{-1}\right)$. The eigenvalues $E$ and $\Lambda$ are related as follows:

$$
E=\Lambda+\nu(\gamma) \cos \theta
$$

It is proved in reference [3] that the functions $\Psi_{\gamma}=e^{2 \pi i(\gamma, w)} f_{\gamma}(z)$ form a complete orthogonal basis on the Sol manifold. Also it is found that the spectrum of the Laplace-Beltrami operator consists of two parts:

- The trivial part, with eigenvalues $E_{k}=\frac{4 \pi^{2} k^{2}}{R^{2}}$, corresponding to $\gamma=0$ in the dual lattice and to eigenfunctions $e^{i 2 \pi k z / R}, k=0,1, \ldots$

- The non-trivial part with eigenvalues $E_{k,[\gamma]}=\Lambda_{k}(\nu[\gamma])+\nu([\gamma]) \cos \theta$ and eigenfunctions the solutions of (28).

In order to compute corrections to the gravitational potential due to Sol structure of extra dimensions, we must compute the first eigenvalues and eigenfunctions. We shall use the most interesting case which is when $\operatorname{Vol}\left(M_{A}^{3}\right) \sin \theta$ is large (or equivalently $\nu \rightarrow 0$ ). Bear in mind that this term contains the compactification radius of the extra dimensions (we must state that the radii of all extra dimensions shall be considered equal) and the deformation of the lattice in terms of $\theta$. Following [3] when $\operatorname{Vol}\left(M_{A}^{3}\right) \sin \theta$ becomes large, $\nu(\gamma)$ becomes small. In that limit the eigenvalues of the Laplace-Beltrami operator, stemming from the non-trivial part, are $E_{k}=\Lambda_{k}$, with $\Lambda_{k}$ the eigenvalues of the modified Mathieu operator,

$$
M=-\frac{d^{2}}{d z^{2}}+|\nu(\gamma)| \cosh 2 \mu z
$$

We order the first eigenvalues as $E_{0}=0 \leq E_{1} \leq E_{2} \leq \ldots$. We shall use the first two, since the corrections to the gravitational potential fall exponentially at the eigenvalues grow larger. Thus the first two are $E_{0}=0$ and $E_{1}$, which asymptotically reads:

$$
E_{1} \sim \frac{(\ln \lambda)^{2} \pi^{2}}{(\ln C)^{2}}
$$


with $C$ as before:

$$
C=\frac{1}{\sqrt{D} \operatorname{Vol}\left(M_{A}^{3}\right) \sin \theta}
$$

The eigenfunctions corresponding to the Mathieu operator (31) are in general,

$$
f_{m}(z)=\sum_{k=0}^{\infty} A_{2 k}^{2 m} \cosh [2 k z]
$$

with ' $m$ ' counting the eigenvalues, $m=0$ corresponds to $E_{0}$ e.t.c.

Now we are ready to compute the modifications to the gravitational potential coming from the first two eigenvalues of the Laplace operator on Sol manifolds. Thus we substitute $E_{0}$ and $E_{1}$ in relation (22) with $\left|\mu_{0}\right|=\sqrt{E_{0}}$ and $\left|\mu_{1}\right|=\sqrt{E_{1}}$. Also we substitute the eigenfunctions for the Sol manifold, thus:

$$
\Psi_{m}(0)=\sum_{k=0}^{\infty} A_{2 k}^{2 m}
$$

The asymptotic behavior of the coefficients $A_{2 k}^{2 m}$ for $\nu \rightarrow 0$ (which is the most interesting case and we shall dwell on it) is really simple [9. It turns out that the terms of the form $A_{m}^{(m)}$ tend to 1 , while terms of the form $A_{m}^{(k)}$, with $m \neq k$ tend to zero. Thus the gravitational potential of relation (22) becomes:

$$
V_{4}=-\frac{G_{4} M}{r} \sum_{m} \sum_{k=0}^{\infty} A_{2 k}^{2 m} A_{2 k}^{2 m} e^{-\left|\mu_{m}\right| r}
$$

So for the first two eigenvalues we have

$$
V_{4}=-\frac{G_{4} M}{r}\left(A_{0}^{0} A_{0}^{0} e^{-\left|E_{0}\right| r}+A_{2}^{2} A_{2}^{2} e^{-\left|E_{1}\right| r}\right)
$$

or (using the asymptotic behavior for the coefficients $A_{m}^{(m)}$ ):

$$
V_{4}=-\frac{G_{4} M}{r}\left(e^{-\sqrt{E_{0}} r}+e^{-\sqrt{E_{1}} r}\right)
$$

Since $E_{0}=0$ and using relations (32) and (33) we obtain finally:

$$
V_{4} \sim-\frac{G_{4} M}{r}\left(1+e^{-\left|\frac{(\ln \lambda) \pi}{(\ln C)}\right| r}\right)
$$

In the relation above it is clear that the Sol manifold correction to the Newton's law gravitational potential depends on the compactification radii of the extra dimensions $R$, the angle $\theta$ of the vectors $e_{u}$ and $e_{v}$ and on the eigenvalues of the hyperbolic gluing map $A$. In the next section we shall investigate the parameter space of the corrections found (which is very rich) and we shall compare it with the $T^{3}$ manifold corrections, to see how much different these are. 


\section{Analysis of the parameter space and comparison with the $T^{3}$ corrections}

The parameter space of the Sol correction to the gravitational potential is very rich. Let us examine first the dependence of the Sol correction range $e^{-\mu_{m} r}$ on the parameters $\theta$ and $n$. In Figure (1) we plot the dependence for the compactification radius value $R=0.05 \mathrm{~mm}$ and for $r=1 \mathrm{~mm}$, while in Figure (2) and (3) the values of $R$ are $0.5 \mathrm{~mm}$ and $1 \mathrm{~mm}$ respectively. We tried to check the plots near the experimental bound for extra dimensions. It seems that the Sol structure gives very large corrections to gravity if the compactification radius is very small (of order $R \sim 10^{-6}$ ). The only interesting cases have compactification radius around $R \sim 1 \mathrm{~mm}$. Looking in Figures (1), (2), and (3), we can see that the last two have similar dependence. It seems that for large values of $n(>200)$ and for $\theta>0.3$, the corrections are very small. In the other two graphs when $n>300$ and for small values of $\theta$, the corrections are small. In the following we shall investigate some interesting cases stemming from the above three. To have a clear picture we shall compare the range of Sol manifolds with the range of the $T^{3}$ torus corrections.

In figure (4) we plot the range of the $T^{3}$ corrections as a function of $r$ with $R=0.05$ and $\theta=\pi / 3$ and in Figure (5) the corresponding dependence for the Sol manifold case. As expected from Figure (1), the term $e^{-\mu_{m} r}$ is very big compared to $e^{-r / R}$, for small $n(\sim 5)$. As $n$ grows the term becomes smaller and smaller. Figure (5) corresponds to $n=250$. As $n$ grows, the range of Sol-corrections becomes comparable and after a value of $n$, smaller compared to the $T^{3}$ corrections. The small $\theta$ case is similar. Concluding the above investigation we could say that the Sol range values are big for the above case in which the corresponding torus range values were small.

Next we shall investigate only the case $R=0.5 \mathrm{~mm}$, since the case $R=1 \mathrm{~mm}$ is similar to it. For the case $\theta=\pi / 3$ and $n=3$, the potential range is exploited in Figure (6) while the case $\theta=\pi / 3$ and $n=300$ in Figure (7). Figure (8) corresponds to the $T^{3}$ case for $R=0.5$. We can see that for small $n$ the Sol corrections are very small while, as $n$ grows, the corrections become three orders smaller, compared to the torus case. Thus Sol corrections can exist for compactification radii and at distances for which the corresponding $T^{3}$ torus range values are very large (and consequently not experimentally preferable).

\section{Conclusions}

In this letter we studied the corrections to the gravitational potential caused by an Sol manifold extra dimensional compact space. Specifically we used the first two eigenvalues of the Laplace-Beltrami operator on the Sol manifold which showed us that the Yukawa type modifications are affected by the parameters $n, \theta$ and $R$, where $R$ the compactification radius and $\theta$ the angle between the basis vectors of the $T^{2}$ lattice. The parameter $n$ is connected with the matrix $A \epsilon S L(2, Z)$, which is hyperbolic and defines the gluing of the torus fibers. After investigating the parameter space we found interesting results, mainly concluding that the range of Sol structure corrections can be similar to the $T^{3}$ torus results 
and (more interestingly) can be very different to the $T^{3}$ results depending to the values of the parameters. This is valuable phenomenologically as can be seen in the previous chapter.

\section{Acknowledgements}

V.O would like to thank Alex Kehagias for reading the manuscript and for valuable comments and prospective extensions of the above he gave.

\section{References}

[1] I. Antoniadis, N. Arkani-Hamed, S. Dimopoulos, G. R. Dvali, Phys. Lett. B436, 257 (1998)

[2] W. P. Thurston, Three-Dimensional geometry and Topology Vol.1, Princeton University Press (1997)

[3] A. V. Bolsinov, H. R. Dullin, A. P. Veselov, Commun. Math. Phys. 264, 583 (2006)

[4] A. Kehagias, K. Sfetsos, Phys. Lett. B472, 39 (2000)

[5] A. Kehagias, J. G. Russo, JHEP, 0007, 027 (2000)

[6] M. Tanimoto, Class. Quant. Grav. 18:479, (2001)

[7] E. G. Floratos, G. K. Leontaris, Phys. Lett. B465, 95 (1999)

[8] H. Kodama, Prog. Theor. Phys. 99, 173 (1998)

[9] N. W. MacLachlan, Theory and Applications of Mathieu Functions, Dover (1964) 


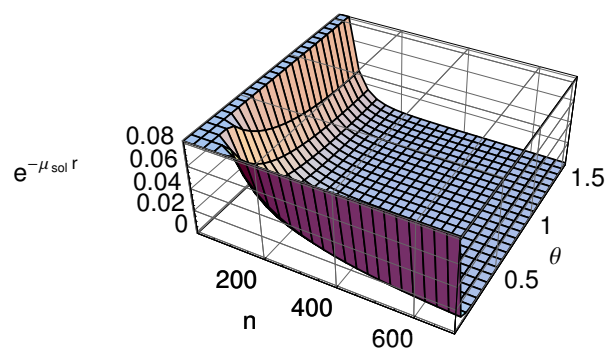

Figure 1: Dependence of the range $e^{-\mu_{m} r}$ of Sol corrections on $\theta$ and $n$ with $r=1 . \mathrm{mm}$, $R=0.05 \mathrm{~mm}$

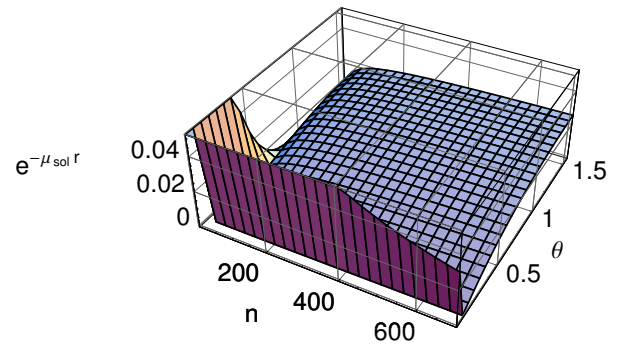

Figure 2: Dependence of the range $e^{-\mu_{m} r}$ of Sol corrections on $\theta$ and $n$ with $r=1 . \mathrm{mm}$, $R=0.5 \mathrm{~mm}$ 


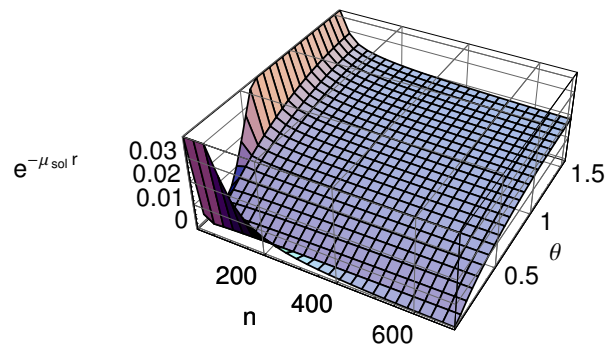

Figure 3: Dependence of the range $e^{-\mu_{m} r}$ of Sol corrections on $\theta$ and $n$ with $r=1 . \mathrm{mm}$, $R=1 \mathrm{~mm}$

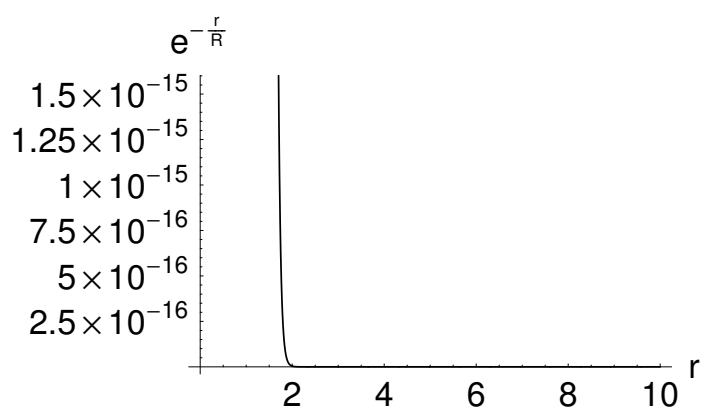

Figure 4: $r$-dependence of the range of $T^{3}$ corrections for $R=0.05 \mathrm{~mm}$

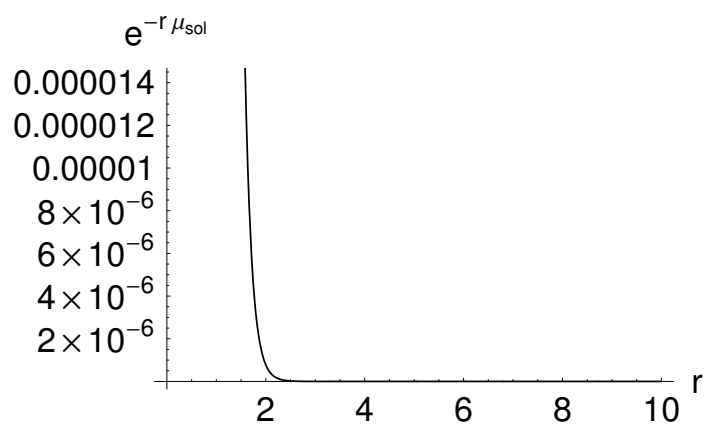

Figure 5: $r$-dependence of the range $e^{-\mu_{m} r}$ of Sol corrections with $R=0.05 \mathrm{~mm}, n=250$ and $\theta=\frac{\pi}{3}$ 


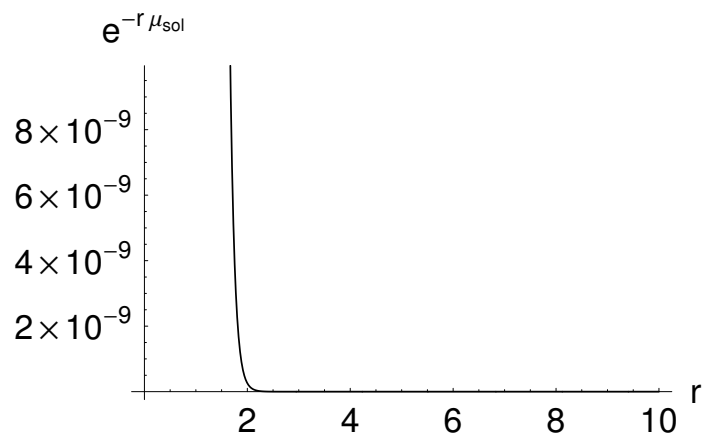

Figure 6: $r$-dependence of the range $e^{-\mu_{m} r}$ of Sol corrections with $n=3, R=0.5 \mathrm{~mm}$ and $\theta=\frac{\pi}{3}$

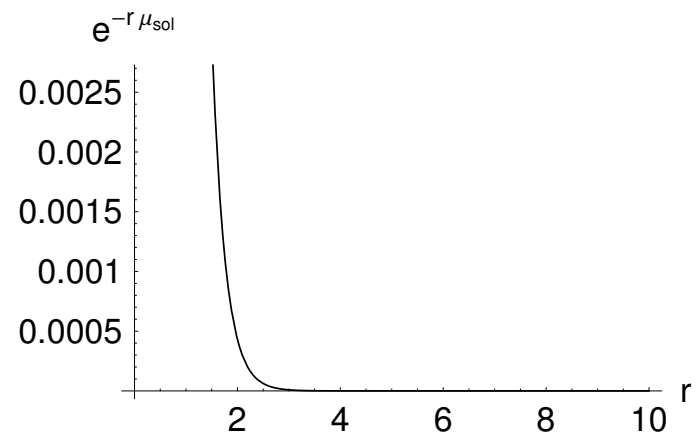

Figure 7: $r$-dependence of the range $e^{-\mu_{m} r}$ of Sol corrections with $n=300, R=0.5 \mathrm{~mm}$ and $\theta=\frac{\pi}{3}$

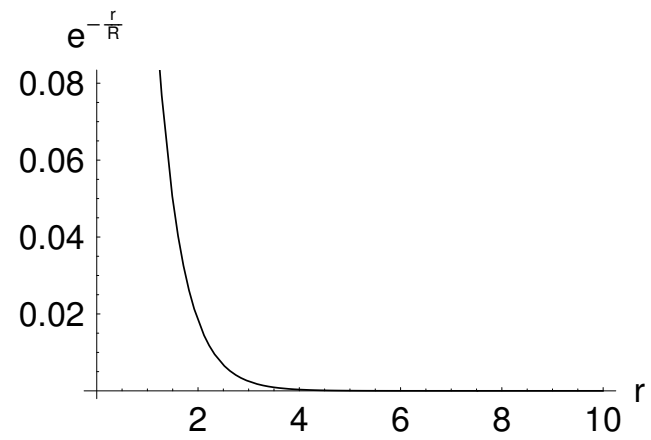

Figure 8: $r$-dependence of the range of $T^{3}$ corrections for $R=0.5 \mathrm{~mm}$ 